COMECHINGONIA. REVISTA DE ARQUEOLOGÍA

Número 14, 2011, pp. 155-172, Córdoba

ISSN 0326-7911

\title{
OCUPACIONES PREHISPÁNICAS EN LA QUEBRADA DE LOS CORRALES, EL INFIERNILLO, TUCUMÁN (ca. 2500-600 AÑOS AP)
}

\section{Nurit Oliszewski ${ }^{1}$}

${ }^{1}$ Instituto Superior de Estudios Sociales (ISES) - CONICET / Universidad Nacional de Tucumán. El Salvador 2190, Yerba Buena, 4107, Tucumán. nuritoli@yahoo.com.ar

Presentado el: 29/12/2010 - Aceptado 5/05/2011

\section{Resumen}

El objetivo del presente trabajo es dar a conocer la secuencia temporal prehispánica de la Quebrada de Los Corrales, ubicada en el abra de El Infiernillo (Tucumán) e insertarla en el marco témporoespacial regional. Tradicionalmente las investigaciones en el NOA se han concentrado en ciertas áreas dejando de lado a otras áreas consideradas marginales como El Infiernillo. Sin embargo, nuestras investigaciones dan cuenta de la presencia de sociedades humanas desde momentos tempranos (anteriores a ca. 2500 años AP) y hasta momentos tardios (ca. 600 años AP). La información generada durante cinco años de trabajo nos lleva a plantear que El Infiernillo y más precisamente la Quebrada de Los Corrales estuvo lejos de tener un carácter marginal en tiempos prehispánicos.

Palabras claves: NOA, El Infiernillo, $1^{\circ}$ milenio D.C., $2^{\circ}$ milenio D.C.

\section{Abstract}

The aim of this paper is to show the time sequence of Quebrada de Los Corrales, located in El Infiernillo (Tucumán) and inserted into the temporal-spatial regional framework. Traditionally, research in the NOA has been concentrated in certain areas, leaving out other areas considered marginal as El Infiernillo. However, our investigations realize the presence of human societies from earliest times (prior to ca. 2500 years BP) until late times (ca. 600 years BP). Information generated during five years of work leads us to propose that El Infiernillo and more precisely Quebrada de Los Corrales was far from a marginal character in ancient times.

Key Words: NWA, El Infiernillo, $1^{\circ}$ millennium A.D., $2^{\circ}$ millennium A.D.

\section{Introducción}

Los primeros intentos por proveer secuencias cronológicas confiables para el NOA (por ejemplo: González 1955, 1979; Núñez Regueiro 1974) no tuvieron en cuenta al abra de El Infiernillo, a pesar de ser ésta una zona de conexión natural entre valles con importantes ocupaciones prehispánicas como Santa María y Amaicha hacia el norte y Tafí hacia el sur. 
Coincido plenamente con Scattolin (2007) en cuanto a que el modelo cultural y cronológico establecido originalmente para otras áreas como Hualfín y Campo del Pucará ha sido erróneamente extrapolado a otras zonas sin tener en cuenta los procesos ocurridos en cada caso particular. De este modo, aún sin ser mencionada explícitamente, el área de El Infiernillo quedó "naturalmente" comprendida dentro de las "secuencias maestras" definidas para otras zonas.

Las investigaciones arqueológicas en el NOA centraron su atención originalmente en ciertas localidades convirtiéndolas en polos del desarrollo prehispánico, mientras otros sectores fueron considerados subsidiarios y/o marginales. En este sentido es emblemático el caso del valle de Tafí -ver Tartusi y Núnez Regueiro (1993)- que ha concentrado por décadas la atención mayoritaria de los investigadores y como consecuencia de ello cuenta con un cúmulo muy grande de información en desmedro de otras áreas. Es este el caso de la zona de El Infiernillo que contaba con un único antecedente bibliográfico, el de Berberián y Giani (2001) quienes planteaban que, debido al escaso desarrollo de los suelos y a su gran altitud, la zona no habría sido apta para actividades agrícolas, aunque sí podrían haber funcionado asentamientos con actividad pastoril intensa. Fue determinante también en el establecimiento de la importancia de unas áreas por sobre otras el desarrollo de las investigaciones anteriores al quehacer científico propiamente dicho (Scattolin 2000).

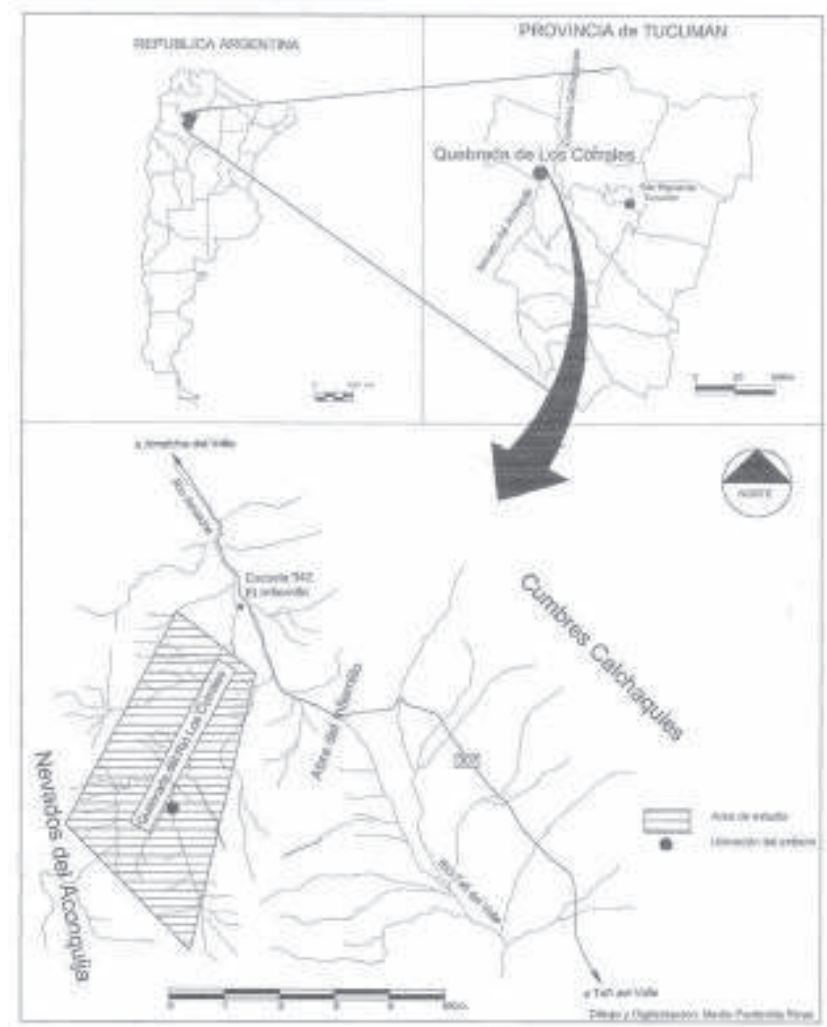

Figura 1. Ubicación del área de estudio. 
Nuestras investigaciones -que se iniciaron en 2005- dan cuenta de la presencia de sociedades humanas desde momentos tempranos (anteriores a ca. 2500 años AP) y hasta momentos tardíos (ca. 600 años AP). El énfasis de estas ocupaciones habría ocurrido durante el $1^{\circ}$ milenio D.C. caracterizado por la asociación de áreas residenciales/domésticas y áreas productivas consistentes en centenares de hectáreas cubiertas por estructuras agrícolas y pastoriles (Caria et al. 2006, 2009, 2010; Oliszewski et al. 2008, 2010a entre otros). La información generada durante cinco años de trabajo nos lleva a plantear que El Infiernillo y más precisamente la Quebrada de Los Corrales estuvo lejos de tener un carácter marginal en tiempos prehispánicos.

En este marco el objetivo del presente trabajo es dar a conocer la secuencia temporal prehispánica de la Quebrada de Los Corrales, ubicada en el abra de El Infiernillo (Tucumán) e insertarla en el marco témporo-espacial regional.

\section{El área de estudio y el estado de la cuestión}

La Quebrada del río de Los Corrales está situada sobre el abra de El Infiernillo (Tucumán), con una cota altimétrica promedio de $3100 \mathrm{msnm}$ y un área total aproximada de $28 \mathrm{~km}^{2}$. Esta quebrada es una zona de hundimiento dentro del sector norte del sistema del Aconquija. Morfológicamente conforma el límite norte del valle de Tafí (Figura1).

Desde 2005 se vienen desarrollando investigaciones arqueológicas sistemáticas que tienen como interés principal profundizar en el conocimiento de las ocupaciones humanas prehispánicas en esta microrregión ${ }^{1}$. Los trabajos de investigación abarcaron la cuenca inferior, media y superior del río de Los Corrales permitiendo identificar hasta el momento dos cuevas con ocupaciones prehispánicas (CC1 y CC2) en la cuenca inferior y media respectivamente, y numerosas estructuras agrícolas, pastoriles y residenciales en la cuenca media/superior (Figura 2).

Cueva de los Corrales 1 (CC1) es una cueva situada en la margen oeste del río de Los Corrales, en la cual se detectó por una parte, una secuencia estratigráfica de $30 \mathrm{~cm}$ de potencia y por otra parte, catorce morteros confeccionados en la roca de base que presentan en casi todos los casos un relleno intencional de restos animales, vegetales y minerales puestos a presión y sellados por el agregado de un sedimento arcilloso. Las excelentes condiciones naturales de preservación permitieron la recuperación de una gran diversidad de restos arqueológicos orgánicos. Dicho contexto permite definir que se trata de un sitio de actividades múltiples, aunque no estrictamente de una vivienda de uso permanente (Oliszewski et al. 2008). Pudieron establecerse las siguientes funciones: procesamiento, consumo y descarte de recursos vegetales alimenticios (Arreguez y González Díaz 2007; Arreguez et al. 2010; Babot 2007; Carrizo et al. 2003; Gramajo Bühler 2007; Oliszewski 2008, 2009); procesamiento, consumo y descarte de recursos animales alimenticios (Srur 2009); producción y aplicación de mezclas pigmentarias empleadas como coberturas cerámicas (Babot y Apella 2007) y producción y mantenimiento de artefactos líticos (Funes Coronel 2007). En cuanto a su ubicación temporal, CC1 presenta dos momentos de ocupación separados por un largo intervalo sin evidencias de uso: las primeras ocupaciones habrían ocurrido hacia ca. 2100 años $\mathrm{AP}$, en momentos agro-pastoriles tempranos, con una ocupación final en momentos tardíos hacia ca. 630 años AP (Oliszewski et al. 2008). 


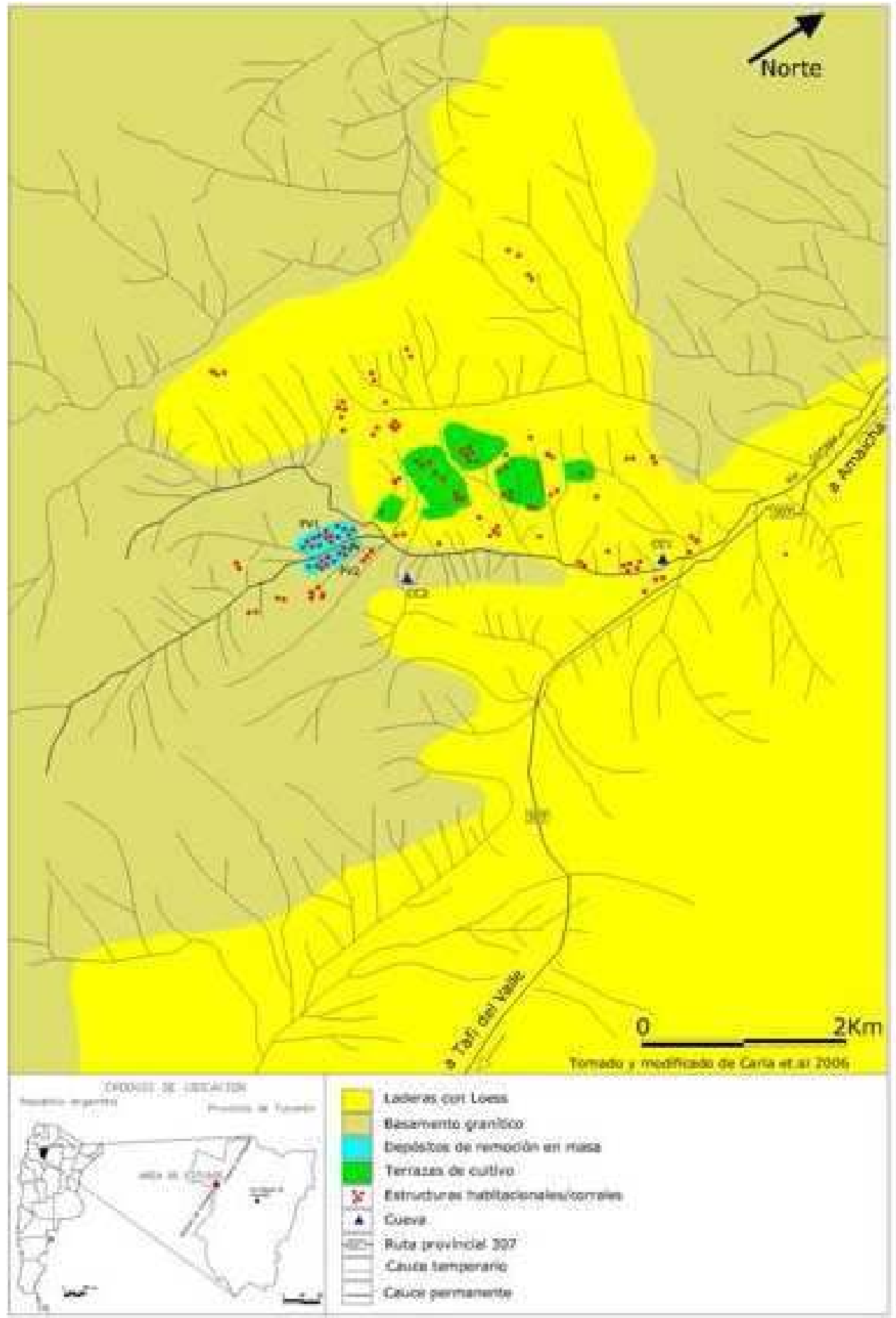

Figura 2. Ubicación de sitios y estructuras arqueológicas en la Quebrada de Los Corrales (modificado de Caria et al. 2006). 
Cueva de los Corrales 2 (CC2) es una pequeña cueva ubicada en la parte superior de un farallón rocoso sobre la margen este del río de Los Corrales. Un sondeo permitió establecer la presencia de dos capas separadas claramente por un hiato estéril. A diferencia de CC1, los hallazgos en ambas capas fueron muy escasos y se limitan a fragmentos óseos de ungulados grandes, lascas de cuarzo y carbones concentrados. CC2 habría estado inicialmente ocupada hacia ca. 1400 años AP relacionada con actividades de caza y/o pastoreo.

Los sistemas de andenería y corrales se ubican en laderas con pendientes de $15^{\circ}$ a $35^{\circ}$ cubriendo una superficie de 500 hectáreas aproximadamente. Cabe aclarar que los sistemas de cultivo no presentan conexión topográfica alguna con el curso fluvial del río de Los Corrales, lo cual permite inferir que este sistema agrícola tuvo como única fuente de riego el manejo del agua de lluvia (Caria et al. 2006, 2009). Respecto a los taxa que podrían haber sido cultivados, se detectó la presencia de fitolitos de poáceas que tienen afinidad con los que se citan para Zea mays L. (Gómez Augier et al. 2008). Este hallazgo es indicativo del posible cultivo de maíz in situ, el cual se registra además en forma de macrorrestos en CC1 y en estratigrafía en una estructura residencial (Oliszewski 2008, 2009; Oliszewski et al. 2010a).

Las estructuras denominadas corrales son aproximadamente 250 y consisten en recintos de planta circular de grandes dimensiones - de entre 20 a $25 \mathrm{~m}$ de diámetro - ya sean individuales o adosados en número de dos o tres.

Las estructuras residenciales, se encuentran concentradas en el área meridional de la Quebrada, en ambas márgenes del curso superior del río de Los Corrales. Los recintos habitacionales ( $\mathrm{N}=52$ ) se presentan como estructuras de piedra subcirculares compuestas Patrón Tafí sensu Berberián y Nielsen (1988)- ubicadas a ca. 3100 msnm (Di Lullo 2009, 2010). Para su estudio esta área ha sido divida en dos sectores: Puesto Viejo 1 (PV1) al oeste y Puesto Viejo 2 (PV2) al este. Entre los materiales recuperados en superficie se puede mencionar abundante material lítico: núcleos y lascas en andesita y cuarzo (Cruz et al. 2009) y material cerámico: fragmentos de diversas facturas, algunos diagnósticos asignables a los estilos cerámicos Tafí, Candelaria, Condorhuasi, Ciénaga y Vaquerías (Gramajo Bühler 2009).

La información generada hasta el momento pone al descubierto una alta variabilidad de evidencias de ocupaciones prehispánicas en la Quebrada de Los Corrales. A partir de esta breve descripción del estado actual de las investigaciones en la microrregión se procederá a continuación a establecer un esquema témporo-espacial de las ocupaciones prehispánicas y una evaluación de los procesos sociales vinculados a dichas ocupaciones.

\section{La secuencia temporal}

El objetivo de este trabajo es, a partir de evidencias artefactuales y arquitectónicas procedentes tanto de superficie como de estratigrafía y dataciones radiocarbónicas en conjunto, establecer una primera aproximación al marco temporal de las ocupaciones prehispánicas en esta microrregión.

No se hará referencia a conceptos como Arcaico, Formativo, Integración Regional o Desarrollos Regionales, ya que considero que se trata de unidades analíticas un tanto rígidas que en la mayoría de los casos no pueden abarcar toda la variabilidad cultural existente. Por otra parte, y como fuera mencionado en la Introducción, estos conceptos que remiten a períodos 
cronológicos con características propias han sido definidos a partir de unos pocos sitios arqueológicos, lo cual puede llevar a que resulten enmascarados los procesos socioculturales locales. En vez de ello se referirá la información generada a tres bloques temporales cuyos límites han sido arbitrariamente establecidos:

1- bloque temporal temprano: anterior al $1^{\circ}$ milenio D.C.,

2-bloque temporal intermedio: $1^{\circ}$ milenio D.C. y

3- bloque temporal tardío: $2^{\circ}$ milenio D.C.

La tabla 1 presenta un detalle de las dataciones radiocarbónicas efectuadas hasta el momento. Las mismas fueron realizadas en el Centro de Estudios Isotópicos Aplicados (CAIS), Universidad de Georgia (EUA). Se consigna, además de la procedencia de cada muestra, el material sobre el que se realizó la datación y el resultado obtenido tanto sin calibrar como calibrado con 1 y 2 sigmas. Las calibraciones fueron realizadas mediante el Programa Calib Radiocarbon Calibration versión 6.0. M. Stuiver, P.J. Reimer, and R. Reimer (http:// calib.qub.ac.uk/calib).

La información proporcionada por los análisis radiocarbónicos más las evidencias artefactuales y arquitectónicas permiten proponer la secuencia cronológica que a continuación se explicita.

1- Bloque temporal temprano: anterior al $^{\circ}$ milenio D.C.

Las evidencias concretas asignables a grupos cazadores-recolectores tempranos (grupos de economía extractiva) para esta zona de valles situados entre 1500 y 3000 msnm era, hasta hace unos años, casi inexistente. El análisis de un conjunto de puntas de proyectil líticas registradas en el área de estudio, más precisamente en la cuenca inferior-media del río de Los Corrales, permitió definir un esquema tipológico-cronológico. Dicho esquema está conformado por al menos tres unidades que exceden la profundidad temporal dada por las dataciones más tempranas para el área de ca. 2100 años AP (Mauri y Martínez 2009). La unidad I (9000?-2500 años AP) está caracterizada por puntas cuyos diseños -triangulares y lanceolados- son idénticos a los recuperados en estratigrafía en sitios arqueológicos de la Puna argentina (Inca Cueva 4 y Peñas de la Cruz 1.1). Los mismos remiten a ocupaciones tempranas de la quebrada, las cuales habrían sido usadas como puntas de proyectiles arrojadizos de sistemas de armas anteriores al uso/aparición del arco y la flecha en el NOA. La Unidad II (2500-1000 años AP) está representada por puntas de proyectil pedunculadas de tamaño pequeño a mediano-pequeño que se adscriben a flechas de arco vinculadas ya a etapas agro-pastoriles ${ }^{2}$. Una tercera unidad (post-1000 años AP) está conformada por puntas de proyectil de tamaño pequeño y de base escotada, que también se corresponderían a sistema de arco y flecha (Mauri y Martínez 2009).

Este esquema cronológico basado en los diseños de las puntas de proyectil pone de manifiesto la probable presencia de grupos humanos con anterioridad a momentos agropastoriles. De hecho, durante 2009 en el sector sur de Puesto Viejo 1 fue detectado próximo a estructuras residenciales, un taller lítico con abundante material en superficie (Taller PV1). Se registraron numerosos desechos de talla y núcleos de andesita y cuarzo (materias primas locales), y también numerosos artefactos formatizados, principalmente puntas de proyectil. Si bien se ubica próximo a estructuras residenciales aldeanas (Puesto Viejo 1), las características técnico-morfológicas de dichas puntas remiten a diseños que podrían 


\begin{tabular}{|c|c|c|c|c|c|c|c|}
\hline $\begin{array}{c}\text { Mevestra } \\
\mathrm{N}^{\prime \prime}\end{array}$ & $\frac{\text { Lab./ }}{\text { Cndige }}$ & $\begin{array}{l}\text { Ideotificación } \\
\text { de la asuestra }\end{array}$ & $\begin{array}{l}\text { Descetipción } \\
\text { de la munestra }\end{array}$ & Añò AP & $\begin{array}{l}\text { Anos } \\
\text { calibraisos } \\
\text { I vigasx } \\
68,306 \\
\text { } 95\end{array}$ & $\begin{array}{l}\text { Anos } \\
\text { calitradus } \\
2 \text { vigman } \\
96,2 \% 0 \\
\text { mci }\end{array}$ & $\frac{A^{x} \times C}{6 \times l}$ \\
\hline 1 & $\begin{array}{l}10 \mathrm{~A} \\
06599\end{array}$ & $\begin{array}{l}\text { QdLC / Sec: } \\
\text { Medsoll E }\end{array}$ & $\begin{array}{l}\text { Sedimento } \\
\text { (estractura } \\
\text { agricola) }\end{array}$ & $500 \pm 30$ & $1313-1357$ & $1205-1370$ & -176 \\
\hline 2 & $\begin{array}{l}\operatorname{voA} \\
0 \operatorname{tog} 7\end{array}$ & $\begin{array}{c}\text { QdLC / CCI. } \\
\text { cap̧a } 1-12^{2} \\
\text { extraccióts) }\end{array}$ & $\begin{array}{l}\text { Poaceas } \\
\text { (camada de } \\
\text { gaja) }\end{array}$ & $630=130$ & $1252-1440$ & $11+5-1524$ & $-24: 1$ \\
\hline 3 & $\begin{array}{l}\text { UGA } \\
\text { OU250 }\end{array}$ & $\begin{array}{l}\text { QdLC / CCL } \\
\text { moutero } 5 \mathrm{bl}\end{array}$ & $\begin{array}{l}\text { Podceas } \\
\text { irallerve de } \\
\text { mortero) }\end{array}$ & $650=30$ & $1339-1357$ & $1343-1394$ & $-23,0$ \\
\hline 4 & $\begin{array}{l}\text { LGA } \\
06796\end{array}$ & $\begin{array}{c}\text { QdLC / CC2 } \\
\text { caps } 2\end{array}$ & Corbodn & $1900=30$ & $622-650$ & 500.008 & -25.2 \\
\hline 5 & $\begin{array}{l}\text { t:GA } \\
\text { O4251 }\end{array}$ & $\begin{array}{l}\text { OdLC/ } \\
\text { Puesto Viejo } 1 . \\
\text { nutrustum } 2\end{array}$ & $\begin{array}{l}\text { Heano } \\
\text { hamasos } \\
\text { Cestherao) }\end{array}$ & $1500=25$ & +35.491 & $427 \cdot 550$ & -184 \\
\hline $\mathrm{b}$ & $\begin{array}{l}176 A \\
06907\end{array}$ & $\begin{array}{l}\text { QdLC / } \\
\text { Puesto Vieja 2, } \\
\text { estroctura 1. } \\
\text { cuadrscula [5, } \\
\text { sural s }\end{array}$ & $\begin{array}{c}\text { Endocerpo } \\
\text { chariar }\end{array}$ & $1000=25$ & $454-532$ & $412-\$ 337$ & -28.4 \\
\hline 7 & $\begin{array}{l}\text { todA } \\
06599\end{array}$ & $\begin{array}{c}\text { QdLC / } \\
\text { Puesto Viejo } 2, \\
\text { estructuin } 1 \text {. } \\
\text { cuadricula H3, } \\
\text { stuvel.13 }\end{array}$ & $\begin{array}{l}\text { Cadikta } \\
\text { vesetal }\end{array}$ & $1090=30$ & $334-400$ & $316-419$ & -268 \\
\hline 8 & $\begin{array}{l}\text { UGA } \\
05093\end{array}$ & $\begin{array}{c}\text { QdLC } \\
\text { Puesto Vieso } 2 . \\
\text { estructurn } 1 . \\
\text { cundisculd Hs, } \\
\text { nuvel } 15\end{array}$ & $\begin{array}{l}\text { Catbat } \\
\text { wegetal }\end{array}$ & $1710=30$ & 322.386 & $253 \cdot 404$ & -23.8 \\
\hline 9 & $\begin{array}{l}\text { viGA } \\
\text { oto16 }\end{array}$ & 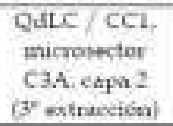 & $\begin{array}{c}\text { Poacens } \\
\text { \{camada do } \\
\text { paja) }\end{array}$ & $2100=200$ & $\begin{array}{l}385 \mathrm{AC} \text { - } \\
\text { 80DC. }\end{array}$ & $\begin{array}{c}\text { 303. A.C. }-342 \\
\text { D.C. }\end{array}$ & .227 \\
\hline
\end{tabular}

Tabla 1. Dataciones radiocarbónicas de la Quebrada de Los Corrales (El Infiernillo, Tucumán). Lab.: laboratorio.

adscribirse al Holoceno Temprano y Medio (ca. 9000 años AP). Las mismas se relacionarían con actividades de caza de grupos que ocuparon la quebrada en momentos previos a la instalación en este sector de grupos agro-pastoriles sedentarios (Martínez comunicación personal).

Las investigaciones a corto plazo en este taller permitirán definir la profundidad temporal de las primeras ocupaciones en la Quebrada de Los Corrales teniendo como hipótesis de trabajo que las mismas serían bastante anteriores a las ocupaciones aldeanas del $1^{\circ}$ milenio D.C.

\section{2- Bloque temporal intermedio: $1^{\circ}$ milenio D.C.}

La datación más temprana de la Quebrada de Los Corrales se encuentra en el límite del $1^{\circ}$ milenio D.C.: $2100 \pm 200$ años AP (UGA 01616) y corresponde a la ocupación más antigua de CC1 (Tabla 1). Esta datación está asociada a actividades de consumo y descarte de recursos alimenticios animales como ungulados grandes (probablemente camélidos) (Srur 2009) y 
vegetales silvestres como algarrobo o chañar y domésticos como maíz (Arreguez et al. 2010; Oliszewski 2009).

Luego se produce un hiato de aproximadamente 400 años ya que, los fechados siguientes corresponden a cuatro dataciones procedentes de capas estratigráficas de recintos habitacionales (Puesto Viejo 1 y 2) acotadas al intervalo ca. 1700 - 1550 años AP. Los primeros datos procedentes de estratigrafía disponibles para una estructura residencial de Puesto Viejo 2 permiten proponer que se trataría de una unidad doméstica en la cual se habrían llevado a cabo diversas actividades cotidianas entre las cuales el consumo y descarte de recursos alimenticios tuvo un papel principal (Oliszewski et al. 2010a). La ocupación inicial habría ocurrido hacia 1700 años AP: $1710 \pm 30$ (UGA 05795) y $1690 \pm 30$ (UGA 06598) y la ocupación final se habría dado hacia 1600 años AP: $1600 \pm 25$ (UGA 06597) (Tabla 1). Las evidencias indican que la estructura habría estado habitada de forma continua a lo largo de un siglo ya que no se registra ningún tipo de diferencias que permitan pensar en dos eventos temporales aislados.

Para Puesto Viejo 1 se cuenta con una única datación realizada sobre material óseo humano procedente de un entierro directo de un individuo masculino adulto (Muntaner 2009; Oliszewski et al. 2010b) la cual arrojó un fechado de $1560 \pm 25$ años AP (UGA 04251) (Tabla 1). El individuo se encontraba acompañado por dos vasijas asignables a los estilos cerámicos Tafí y Candelaria.

Uno de los cuestionamientos inicialmente planteados fue la posible contemporaneidad entre PV1 y PV2. La calibración con 2 sigmas (95,4 \% de probabilidad) de las dataciones de ambos sectores indica, hasta el momento, que podrían haber estado ocupados en simultáneo ya que la única datación de PV1 (427- 557 Cal. D.C.) y la datación más tardía de PV2 (412537 Cal. D.C.) presentan superposición en sus rangos (Tabla 1).

Con posterioridad a ca. 1550 años AP y fuera del ámbito de las estructuras residenciales, una datación procedente de CC2 de $1400 \pm 30$ años AP (UGA 05796), permite proponer a modo de hipótesis que esta ocupación pudo haberse extendido hasta este momento, lo cual deberá ser corroborado en el corto plazo (Tabla 1).

Por el momento podemos afirmar que la ocupación más intensa en la Quebrada de Los Corrales tuvo lugar a mediados del $1^{\circ}$ milenio D.C. en Puesto Viejo y se dio a lo largo de aproximadamente 150 años entre $c a .1700$ y 1550 años AP con probabilidades de haber continuado hasta ca. 1400 años AP.

Un problema de difícil resolución es el de la correlación entre el lapso de ocupación de Puesto Viejo y los sectores productivos tanto agrícolas como pastoriles. Pero, al no contar por el momento con ningún tipo de evidencia que indique lo contrario, creemos que tanto corrales como andenes habrían funcionado durante el $1^{\circ}$ milenio D.C., teniendo como hipótesis que la gente que habitó Puesto Viejo fue la misma que manejó las estructuras productivas de la Quebrada de Los Corrales.

Asumiendo la contemporaneidad de uso de las estructuras domésticas y productivas, es interesante destacar que el manejo del espacio se da en esta quebrada de un modo particular. Los espacios, donde se emplazaron las estructuras productivas, se encuentran totalmente 
separados de aquellos donde se instalaron las estructuras residenciales. Las estructuras de producción agrícola (andenes) y la mayoría de los corrales se ubican sobre laderas con depósitos loéssicos, mientras que las estructuras residenciales se sitúan sobre depósitos de remoción en masa y en laderas con sustrato de basamento granitoide, delimitando espacios diferenciados entre sí (Caria et al. 2006, 2009). Coincido con Di Lullo (2010) en cuánto a que más allá de las diferencias geolitológicas de los lugares de emplazamiento de las estructuras productivas y domésticas, estas sociedades habrían incluido sus conceptos de lo "doméstico" y lo "productivo" en campos semánticos distintos y/u opuestos, y que esto se vio reflejado en el modo de estructurar el espacio.

La disposición espacial de las unidades habitacionales y de las estructuras agrícolas y pastoriles, las evidencias artefactuales y las dataciones absolutas demuestran que durante la primera mitad del $1^{\circ}$ milenio D.C. existió una aldea ubicada a $c a .3100 \mathrm{msnm}$, en una quebrada de altura a la cual hasta el momento no se le había dado la misma importancia que a los valles aledaños de Santa María y Tafí.

\section{3- Bloque temporal tardío: $2^{\circ}$ milenio D.C.}

A diferencia de lo que ocurre para $\mathrm{el} 1^{\circ}$ milenio donde hay numerosos elementos como estructuras residenciales, estructuras productivas, estilos cerámicos o puntas de proyectil que permiten proponer una ocupación sostenida e intensa, para el $2^{\circ}$ milenio las evidencias

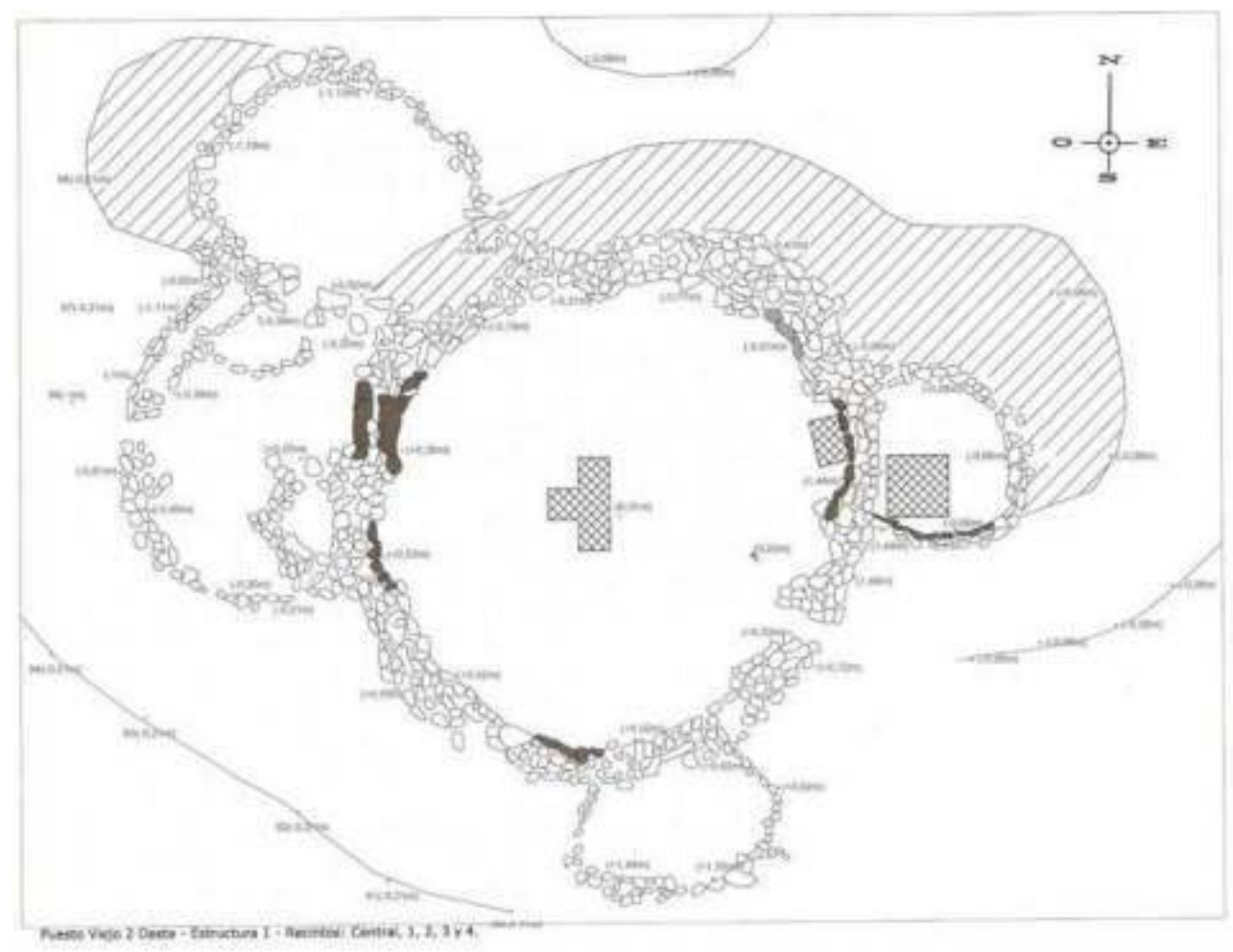

Figura 3. Dibujo de planta de una estructura de Puesto Viejo. 
para referirse a una ocupación constante son sumamente escasas. Las mismas se reducen a tres fechados radiocarbónicos, dos de los cuales provienen de CC1: uno $-630 \pm 140$ años AP (UGA 01977) - de la capa estratigráfica $1^{\circ}$ ( $2^{\circ}$ extracción) y otro $-650 \pm 30$ años AP (UGA 04250)- de uno de los morteros que conecta con la capa mencionada (Tabla 1). Cabe destacar que no se han registrado ni en capa ni en el mortero fragmentos cerámicos asignables a estilos tardíos pero tampoco a estilos tempranos del $1^{\circ}$ milenio D.C. como los hallados en el resto de la Quebrada de Los Corrales. Ambas dataciones estarían marcando el fin de las ocupaciones de CC1, que no habrían sido ininterrumpidas sino que, entre la ocupación más temprana ocurrida hacia 2100 años AP y ésta última habría un largo intervalo sin evidencias de ocupación.

El tercer fechado se realizó sobre sedimento procedente de un andén de cultivo dando como resultado $590 \pm 30$ años AP (UGA 06599) (Tabla 1). Dicho sedimento -situado a $30 \mathrm{~cm}$ de profundidad- habría constituido el "suelo" donde se cultivó en momentos prehispánicos. En apoyo de esta hipótesis se registraron en el sedimento datado y en otras 3 muestras de sedimento procedentes de andenes similares fitolitos afines a Zea mays (Gómez Augier et al. 2008). De todos modos esta datación no invalida la hipótesis de que las estructuras agrícolas habrían funcionado durante el $1^{\circ}$ milenio D.C. en vinculación con el sector residencial (Puesto Viejo) por los siguientes motivos:

a) la datación proviene de un sector de andenes atípicos ya que están asociados a un conjunto compuesto por siete escalones de piedra y dos estructuras monticulares también de piedra que al parecer habrían tenido un carácter ceremonial;

b) podría tratarse del último uso de la estructura agrícola que probablemente habría sido utilizada desde tiempos anteriores.

Es decir que, más allá de estas dataciones, no hay indicios claros de ocupaciones tardías como podría ser la presencia de casas-pozo de planta cuadrangular y/o cerámica "tardía" tipo santamariana, ítems que se vinculan, sin dudas, a sociedades del $2^{\circ}$ milenio D.C. dentro de los sectores adyacentes a esta área.

\section{Comentarios}

Sobre nueve dataciones realizadas se observa:

1) un fechado inicial aislado de ca. 2100 años AP en un contexto de cueva (CC1).

2) un agrupamiento de cuatro dataciones en un intervalo que abarca 150 años aproximadamente (ca.1700 - 1550 años AP) lo cual está apoyado por el registro de estructuras domésticas/residenciales conformando una aldea (PV1 y PV2), materiales cerámicos (estilos Vaquerías, Condorhuasi, Ciénaga, Candelaria y Tafí) y materiales líticos (puntas de proyectil) característicos de este momento.

3) un fechado de ca. 1400 años AP en contexto de cueva (CC2) que se presume podría estar relacionado con los cuatro fechados anteriores.

4) un agrupamiento de tres dataciones entre $c a .650$ y 590 años AP en un intervalo tardío que pueden considerarse contemporáneos. No está soportado por la presencia de evidencias inmuebles características de este período para áreas aledañas como estructuras residenciales o defensivas (fuertes). Tampoco se observan materiales cerámicos característicos de momentos tardíos, aunque sí algunas puntas de proyectil halladas en superficie. 
La ocupación más intensa habría ocurrido durante el $1^{\circ}$ milenio D.C. más precisamente en la primera mitad. Se propone a modo de hipótesis que las ocupaciones anteriores estarían marcando una continuidad desde antes de ca. 2500 años AP. En cambio la ocupación final, posterior a ca. 600 años AP no tendría relación con los grupos anteriores. Se propone también a modo de hipótesis un quiebre en las ocupaciones de la Quebrada con posterioridad a ca. 1500 años AP por motivos que habrá que dilucidar. La última ocupación habría sido muy esporádica, no teniendo la duración temporal, ni la intensidad de las ocupaciones anteriores.

\section{La Quebrada de Los Corrales en el marco regional}

Una vez presentada la secuencia temporal de la Quebrada de Los Corrales se vuelve necesario indagar acerca de cómo y cuándo fueron ocupados los espacios de los valles próximos: Santa María y Amaicha hacia el norte y Tafí hacia el sur.

Las dataciones más antiguas rondan los 2500 años AP tanto para el valle de Santa María (Scattolin 2007) como para el valle de Tafí (Sampietro y Vattuone 2005). La presencia en la Quebrada de Los Corrales de puntas de proyectil que serían previas a este momento invitan a indagar con mayor profundidad acerca de las primeras ocupaciones a nivel regional. Pero, cabe destacar que, las primeras ocupaciones para la región pueden haber sido mucho más tempranas aún, tal como lo demuestran investigaciones realizadas por Somonte (2009) en Amaicha del Valle (sitio Planchada La Puntilla) mediante la técnica VML (varnish microlamination, sensu Liu y Dorn 1996) que dan cuenta de nuevas evidencias datadas hacia el Holoceno Medio (7300-5900 AP) y por Mauri y Martínez (2009) en la Quebrada de Los Corrales que describieron puntas de proyectil con diseños asignables al Holoceno Temprano y Medio.

En cuanto al $1^{\circ}$ milenio son numerosos los sitios arqueológicos asignables a este período caracterizados por su patrón de asentamiento de tipo aldeano agrupado o "Patrón Tafí": habitaciones circulares concentradas en torno a patios centrales y asociadas a estructuras agrícolas y corrales. Si bien el patrón es semejante al de nuestro caso de estudio, en general las estructuras residenciales y productivas se encuentran asociadas espacialmente a diferencia de lo que ocurre en la Quebrada de Los Corrales donde ambos tipos de estructuras se encuentran claramente separadas entre sí. Retomando la idea de lo doméstico y lo productivo, Di Lullo (2010) propone para la Quebrada de Los Corrales que la sociedad percibía a las actividades domésticas y agro-pastoriles en campos semánticos diferentes. Coincido con la autora en que esta percepción semántica diferencial habría tenido su correlato en la estructuración del espacio lo cual involucraba a las nociones de "área residencial" y "área productiva" como opuestas pero al mismo tiempo en constante interacción.

En todos los casos es recurrente la presencia de estilos cerámicos como Tafí, Candelaria, Condorhuasi, Ciénaga y Vaquerías. Es interesante hacer notar que tradicionalmente cada uno de estos estilos cerámicos estuvo vinculado a un área específica como, por ejemplo, Tafí al valle homónimo o Candelaria al piedemonte salteño-tucumano. El devenir de las investigaciones ha ido revirtiendo esto, siendo actualmente uno de los objetivos dilucidar el por qué de la presencia de todos los estilos en todas partes al mismo tiempo.

La gran mayoría de los sitios asignables al $1^{\circ}$ milenio D.C. se encuentran en el valle de Tafí: Casas Viejas (Núñez Regueiro y García Azcárate 1996), Casa Machado (Chocobar y 
Corbalán 2005) y El Potrerillo (Gómez Cardozo 2001) en las cercanías de El Mollar; El Tolar en el cono del río Blanco (Sampietro y Vattuone 2005); El Pedregal (Cremonte 1996) en el valle de la Ciénega y La Bolsa (Giani y Berberián 1999; Salazar y Franco Salvi 2009). Pero también hay algunos sitios en el valle de Amaicha como Campo Blanco (Somonte 2002) y El Remate (Aschero y Ribotta 2007) y en el valle de Santa María como Bañado Viejo (Scattolin et al. 2001). Todos ellos ponen de manifiesto que, al igual que en la Quebrada de Los Corrales, las ocupaciones durante el $1^{\circ}$ milenio habrían sido intensas y sostenidas a lo largo de cientos de años.

Las evidencias asociadas al $2^{\circ}$ milenio D.C. están representadas por núcleos residenciales de complejidad semiurbana vinculados a estructuras productivas agrícolas y ganaderas que denotan una intensificación del uso respecto a momentos anteriores. Es característico el registro de cerámica estilo Santa María y artefactos de metal relacionados con actividades como la guerra y la muerte. Este tipo de evidencias son numerosas en el valle de Santa María -El Pichao (Medina y Cornell, 2011) y Quilmes (Pelissero y Difrieri 1981)- son dos claros ejemplos, existiendo algunos sitios en el valle de Amaicha como El Observatorio (Gómez Augier 2005) y Los Cardones (Rivolta 2007). No ocurre lo mismo en el valle de Tafí donde las evidencias asignables al $2^{\circ}$ milenio D.C. son mucho más escasas que las del milenio anterior. No se encuentran los grandes y complejos núcleos residenciales típicos del valle de Santa María pero sí se registran algunas casas-pozo de planta cuadrangular características de Santa María junto a recintos que presentan un patrón de tipo aldeano agrupado (Núñez Regueiro y Esparrica 2001). También se registra en algunos sitios cerámica Santa María como en La Costa 1 (Manasse 2007) o el mencionado El Pedregal en el valle de La Ciénega (Cremonte 1996). Al parecer las ocupaciones no habrían sido tan intensas pero, me parece totalmente acertada la observación de Manasse (2007) quien afirma que las investigaciones en el valle se concentraron en los momentos tempranos, siendo pocas aquellas que investigan la presencia de grupos durante el $2^{\circ}$ milenio D.C.

En la Quebrada de Los Corrales -si bien se cuenta con tres dataciones asociadas a ca. 600 años AP- no hay indicios de patrones de asentamiento ni presencia de los clásicos estilos cerámicos relacionados a momentos tardíos como el estilo Santa María tal como ocurre en el valle de Tafí. Futuras investigaciones permitirán aclarar tanto la naturaleza de las últimas ocupaciones como su relación con los valles situados al norte y al sur de la misma.

En síntesis la Quebrada de Los Corrales se encuentra en consonancia con el panorama regional: escasos pero significativos indicios de ocupaciones anteriores a 2500 años AP que llevan a preguntarse no sólo por los primeros habitantes sino por la relación de estos con los grupos que vendrían a continuación; ocupaciones continuadas e intensas durante el $1^{\circ}$ milenio D.C. y ocupaciones más efímeras durante el $2^{\circ}$ milenio D.C.

Para terminar, me parecen pertinentes las preguntas planteadas por Laguens (2007) acerca de si hubo coexistencia de diferentes grupos en diferentes lugares, inclusive muy próximos, que aparentemente no habrían interaccionado entre ellos y si debemos pensar a los desarrollos locales como procesos independientes.

La información hasta ahora generada apunta a una respuesta intermedia: pienso que cada grupo tuvo su carácter distintivo y por lo tanto siguió sus propios procesos históricos pero que seguramente deben haber interaccionado en forma fluida entre ellos. Si bien hay 
elementos comunes en muchos de estos sitios como es el caso para el $1^{\circ}$ milenio D.C. del patrón de asentamiento aldeano agrupado o la presencia de estilos cerámicos como Candelaria, Tafí, Condorhuasi, Ciénaga o Vaquerías, también es cierto que cada grupo social parece haber tenido ciertas particularidades que lo volvían único. Consideramos que es éste el caso de la Quebrada de Los Corrales dónde en un momento temprano (ca. 1700 años AP) cuando lo esperable es que las viviendas se encuentren dispersas entre las estructuras productivas -según el modelo propuesto por Berberián y Nielsen (1988)-, ocurre lo contrario: estructuras residenciales y productivas se encuentran claramente separadas espacialmente. Esto lleva a pensar acerca de si la explicación se puede buscar en la situación topográfica particular de tratarse de una quebrada y no de un valle o si es la idiosincrasia del grupo y no la topografía la que determinó que la esfera doméstica y la esfera productiva se encuentren separadas entre sí. En este sentido retomo la idea de Laguens (2007) acerca de que el tiempo toma sentido al constituirse en el tiempo de los individuos y de la sociedad. Es decir que cada sociedad tuvo su propia lógica en cada momento particular.

Es de esperar que a este intento de mostrar que las mal llamadas "áreas marginales" no fueron tales sino que por el contrario, formaron parte de una intrincada red de grupos sociales que, al mismo tiempo, presentaban características particulares, se sumen otros que den cuenta del complejo esquema social, temporal y espacial que caracterizó al Noroeste argentino en tiempos prehispánicos.

\section{Agradecimientos}

Las investigaciones se subsidian con subsidios de ANPCyT y CIUNT. Muchas gracias a todos los miembros del equipo de trabajo. Un especial agradecimiento a Mario Caria y Jorge Martínez.

\section{Notas}

${ }^{1}$ Las investigaciones se implementaron en el marco de proyectos subsidiados por el Consejo de Investigaciones de la Universidad Nacional de Tucumán (26/G318 y 26/G433) y la Agencia Nacional de Promoción Científica y Tecnológica (PICT-2006-01245).

${ }^{2}$ La ubicación cronológica de los diseños correspondientes al lapso -ca. 2500 - 1000 años AP- proviene de puntas de proyectil recuperadas en estratigrafía en sitios de la Puna Meridional (Martínez, comunicación personal).

\section{Bibliografía citada}

Aschero, C.A. y E.E. Ribotta

2007. Usos del espacio, tiempo y funebria en el remate (Los Zazos, Amaicha del Valle, Tucumán). Paisajes y procesos sociales en Tafi del Valle (comp. por P. Arenas, B. Manasse y E. Noli), pp. 79-94. V. Ataliva editor, Tucumán.

Arreguez G.A. y L.N. González Díaz

2007. Uso de recursos vegetales en un sitio prehispánico de altura: Cueva de los Corrales 1 (El Infiernillo, Tafí del Valle, Tucumán). Primeras Jornadas de Jóvenes Investigadores UNT-AUGM. Consejo de Investigaciones de la Universidad Nacional de Tucumán, Tucumán. 
Arreguez, G.A.; Gramajo Bühler, C.M.y N. Oliszewski

2010. Utilización de recursos vegetales alimenticios en sitios arqueológicos de altura. El caso de Cueva de Los Corrales 1 (El Infiernillo, Tafí Del Valle, Tucumán, Argentina). La arqueometría en Argentina y Latinoamérica (ed. por G.R. Cattáneo, A.D. Izeta y G. Castellano), Editorial de la FFyH, Universidad Nacional de Córdoba, Córdoba (en prensa).

Babot, M.P.

2007. Granos de almidón en contextos arqueológicos: posibilidades y perspectivas a partir de casos del Noroeste argentino. Investigaciones arqueobotánicas en Latinoamérica: estudios de casos y propuestas metodológicas (comp. por M.B. Marconetto, M.P. Babot y N. Oliszewski), pp. 95-125. Centro Editorial de la Facultad de Filosofía y Humanidades, Universidad Nacional de Córdoba, Ferreira editor, Córdoba.

Babot, M.P. y M.C. Apella

2007. Aproximación al proceso de producción de alfarería en el Área Valliserrana de Tucumán, Argentina: un análisis de mezclas pigmentarias y coberturas cerámicas. Cerámicas arqueológicas. Perspectivas arqueométricas para su análisis e interpretación (ed. por B. Cremonte y N. Ratto), pp. 13-26. Editorial de la Universidad Nacional de Jujuy, San Salvador de Jujuy.

Berberián, E.E. y L. Giani

2001. Organización intrasitio y macroespacial en el Formativo del valle de Tafí (Tucumán). Actas del XIII Congreso Nacional de Arqueología Argentina, Tomo I, pp. 409-415. Editorial Brujas, Córdoba.

Berberián, E.E. y A. Nielsen.

1988. Sistemas de asentamiento prehispánicos en la etapa Formativa del Valle de Tafí (Pcia. de Tucumán - República Argentina). Sistemas de asentamiento prehispánicos en el Valle de Tafí (ed. por E.E. Berberián), pp. 21-51. Córdoba.

Caria, M.A.; Martínez, J.G. y N. Oliszewski

2006. Geomorfología y Arqueología de la Quebrada del Río de los Corrales (El

Infiernillo-Tafí del Valle-Tucumán-Argentina). Actas de Trabajos del III

Congreso Argentino de Cuaternario y Geomorfología (ed. por Sanabria y Argüello), Tomo I, pp. 145-154. Universidad Nacional de Córdoba, Córdoba.

2009. Los geoespacios arqueológicos durante el Holoceno Superior en la Quebrada del río de Los Corrales (El Infiernillo, Tucumán, Argentina). Geomorfología y cambio climático (ed. por J.M. Sayago y M. Collantes), pp. 145-162. Instituto de Geociencias y medioambiente (INGEMA), Universidad Nacional de Tucumán, Tucumán.

Caria, M.A.; Oliszewski, N.; Gómez Augier, J.P.; Pantorrilla, M.M. y Gramajo Bühler, C.M. 2010. Formas y espacios de las estructuras agrícolas prehispánicas en la Quebrada del río de Los Corrales (El Infiernillo-Tucuman). Arqueología de la agricultura: casos de estudio en la región andina argentina (ed. por M.A. Korstanje y M. Quesada), pp. 144-165 (en prensa).

Carrizo, J.I.; N. Oliszewski y J.G. Martínez

2003. Macrorrestos vegetales del sitio arqueológico Cueva de los Corrales (El Infiernillo, Tafí del Valle, Tucumán). Revista del Museo Argentino de Ciencias Naturales. n.s. 5 (2): 253-260. 
Cremonte, M. B.

1996. Investigaciones Arqueológicas en la Quebrada de la Cienaga (Dpto. Tafí, Tucumán). Tesis Doctoral. Universidad Nacional de La Plata, Buenos Aires.

Cruz, H.; Mauri, E.P. y J.G. Martínez.

2009. Reconocimiento de fuentes de aprovisionamiento prehispánicas de materias primas líticas en la Quebrada de Los Corrales (El Infiernillo, Tucumán, Argentina). Serie Monográfica y Didáctica 48: 111. Facultad de Ciencias Naturales e Instituto Miguel Lillo, Universidad Nacional de Tucumán, Tucumán.

Chocobar, M.F. y M.H. Corbalán

2005. Las estructuras arqueológicas olvidadas de Casa Machado. Serie Monográfica y Didáctica 45: 13. Facultad de Ciencias Naturales e Instituto Miguel Lillo, Universidad Nacional de Tucumán, Tucumán.

Di Lullo, E.

2009. Mapeo de recintos habitacionales en la Quebrada de Los Corrales (El Infiernillo, Tucumán). Serie Monográfica y Didáctica. 48: 115. Facultad de Ciencias Naturales e Instituto Miguel Lillo, Universidad Nacional de Tucumán, Tucumán.

2010. El espacio residencial durante el 1er milenio d. C. en la Quebrada de Los Corrales (El Infiernillo, Tucumán). Tesis de Grado en Arqueología. Facultad de Ciencias Naturales e Instituto Miguel Lilo, Universidad Nacional de Tucumán. Tucumán.

Funes Coronel, J.A.

2007. Caracterización del conjunto lítico del sitio Cueva de Los Corrales 1 (CC1), El Infiernillo, Tucumán. Primeras Jornadas de Jóvenes Investigadores UNT-AUGM. Consejo de Investigaciones de la Universidad Nacional de Tucumán, Tucumán.

Giani, L. y E.E. Berberián

1999. Consideraciones acerca de la variabilidad formal en el diseño de las plantas de arquitectura en el NOA durante las etapas Formativa y de Desarrollos Regionales. Actas del XII Congreso Nacional de Arqueología Argentina, Tomo I, pp. 83-88, Universidad Nacional de La Plata, La Plata.

Gómez Augier, J.P.

2005. Geoarqueología y patrones de ocupación espacial en el sitio El Observatorio. Ampimpa, Dpto. Tafí del Valle, Tucumán, República Argentina. Tesis de Grado en Arqueología. Facultad de Ciencias Naturales e Instituto Miguel Lilo, Universidad Nacional de Tucumán. Tucumán.

Gómez Augier, J.P.; Oliszewski, N. y M.A. Caria.

2008. Altitude cultivation: phytolith analysis in archaeological farming structure of Quebrada del Río de Los Corrales site (El Infiernillo, Tucumán, Argentina). International Meeting on Phytolith Research. 4th Southamerican Meeting Phytolith Research. Abstracts (ed. por M. Osterrieth, Fernández Onaire y Borelli), pp. 64, Mar del Plata.

Gómez Cardozo, C.

2001. Pastoreo de camélidos en el Valle de Tafí (Provincia de Tucumán, Argentina). XIV Congreso Nacional de Arqueología Argentina, Libro de resúmenes, pp. 436. Universidad Nacional de Rosario, Rosario, 
González, A.R.

1955. Contextos culturales y cronología relativa en el área central del N. O. argentino (nota preliminar). Anales de Arqueología y Etnología 11: 7-32.

1979 Dinámica cultural del N.O. argentino. Evolución e historia en las culturas del N.O. argentino. Antiquitas. Boletín de la Asociación Amiga del Instituto de Arqueología 28-29: 115.

Gramajo Bühler, C.M.

2007. Caracterización y descripción taxonómica del contenido del mortero 5 b1 del sitio Cueva de Los Corrales 1. Serie Monográfica y Didáctica. 46:35. Facultad de Ciencias Naturales e Instituto Miguel Lillo, Universidad Nacional de Tucumán, Tucumán.

2009. Primera caracterización del conjunto cerámico de la Quebrada de Los Corrales (El Infiernillo, Tucumán). Serie Monográfica y Didáctica. 48: 121. Facultad de Ciencias Naturales e Instituto Miguel Lillo, Universidad Nacional de Tucumán, Tucumán.

Laguens, A. G.

2007. Tiempo y cronologías en la arqueología del NOA. Sociedades precolombinas surandinas: Temporalidad, interacción y dinámica cultural del NOA en el ámbito de los Andes Centro-Sur (ed. por V. Williams, B. Ventura, A. Callegari y H. Yacobaccio), pp. 287-297. Artes Gráficas Buschi, Buenos Aires.

Liu, T. y Y. Dorn.

1996. Understanding Spatial Variability in Environmental Changes in Drylands with Rock Varnish Microlaminations. Annals of the Association of American Geographers 86:187-212.

Manasse, B.

2007. Tiempo antes de la conquista española en el Valle de Tafí ... Paisajes y procesos sociales en Tafi del Valle (comp. por P. Arenas, B. Manasse y E. Noli), pp. 135-164. V. Ataliva editor, Tucumán.

Mauri, P. E. y J. G. Martínez.

2009. Análisis de puntas de proyectil líticas de la Quebrada de Los Corrales (El Infiernillo, Tucumán) y sus implicancias tecno-tipológicas y cronológicas. Serie Monográfica y Didáctica. 48: 131. Facultad de Ciencias Naturales e Instituto Miguel Lillo, Universidad Nacional de Tucumán, Tucumán.

Medina, M.C. y P. Cornell

2011. El Proyecto Pichao 1989-2005: apuntes de su historia y resultados. Rastros en el camino... Trayectos e identidades de una institución. Homenaje a los 80 años del IAM-UNT, pp. 245-255. Editorial EDUNT, Tucumán

Muntaner, A.C.

2009. Identificación anatómica y Estimación de sexo, edad y estatura de un enterratorio humano en la quebrada de los corrales (El Infiernillo, Tucumán). Serie Monográfica y Didáctica. 48: 145. Facultad de Ciencias Naturales e Instituto Miguel Lillo, Universidad Nacional de Tucumán, Tucumán. 
Núñez Regueiro, V.A.

1974. Conceptos instrumentales y marco teórico en relación al análisis del desarrollo cultural del Noroeste argentino. Revista del Instituto de Antropología, Universidad Nacional de Córdoba 5: 168-190.

Núñez Regueiro, V.A. y H. Esparrica.

2001. Investigaciones arqueológicas en la zona del $\mathrm{km} 64,5$, Valle de Tafí, Provincia de Tucumán. XIV Congreso Nacional de Arqueología Argentina, Libro de resúmenes, pp. 167-168. Universidad Nacional de Rosario, Rosario.

Núñez Regueiro, V.A. y J. García Azcárate.

1996. Investigaciones arqueológicas en El Mollar, Dpto. tafí del Valle, Pcia. de Tucumán. Revista del Museo de Historia Natural de San Rafael XXV, 1/2: 87-98.

Oliszewski, N.

2008. Metodología para la identificación subespecífica de maíces arqueológicos. Un caso de aplicación en el noroeste de argentina. Arqueobotánica y Teoría Arqueológica. Discusiones desde Suramérica (ed. por S. Archila, M. Giovannetti y V. Lema), pp. 181-202. Uniandes- Ceso, Bogotá.

2009. El recurso maíz en sitios arqueológicos del noroeste argentino: el caso de la Quebrada de Los Corrales, El Infiernillo, Tucumán. Treballs d'Etnoarqueologia 7: 83-96, Madrid.

Oliszewski, N.; Arreguez, G.A., Cruz, H.; Di Lullo, E.; Gramajo Bühler, C.M.; Mauri, E.P; Pantorrilla Rivas, M.M. y M.G. Srur.

2010a. Puesto Viejo: una aldea temprana en la Quebrada de Los Corrales (El Infiernillo, Tucumán). Actas del XVII Congreso Nacional de Arqueología Argentina, Tomo IV, pp. 1697-1702.

Oliszewski, N.; Gramajo Bühler, C.M.; Mauri, E.P.; Miguez, G.E.; Muntaner, A.C. y M.M. Pantorilla Rivas

2010b. Caracterización de un enterratorio humano en la Quebrada de Los Corrales (El Infiernillo, Tucumán). Intersecciones en Antropología 11:315-319.

Oliszewski, N.; Martínez, J.G. y M.A. Caria

2008. Ocupaciones prehispánicas de altura: el caso de Cueva de los Corrales 1 (El Infiernillo, Tafí del Valle, Tucumán). Relaciones de la Sociedad Argentina de Antropología XXXIII: 209-221.

Pelissero, N.A. y H.A. Difrieri

1981. Quilmes. Gobierno de la Provincia de Tucumán, UBA, Tucumán.

Rivolta, G.

2007. Diversidad cronológica y estructural en los diferentes sectores de la Quebrada de Los Cardones: sus espacios y recintos (Valle de Yocavil, Tucumán). ). Paisajes y procesos sociales en Tafí del Valle (comp. por P. Arenas, B. Manasse y E. Noli), pp. 95-110. V. Ataliva editor, Tucumán.

Salazar, J. y V.L. Franco Salvi.

2009. Una mirada a los entornos construidos en el valle de Tafí, Tucumán (1 - 1000 AD). Comechingonia 12: 91-108. 
Sampietro, M. y M. Vattuone

2005. Reconstruction of activity areas in northwest Argentina. Geoarchaeology: An International Journal, Vol. XX, No. 4: 337-354.

Scattolin, M.C.

2000. Santa María durante el primer milenio DC. ¿Tierra baldía? Årstryck 1995-1998: 63-83. 2007. Santa María antes del año mil. Fechas y materiales para una historia cultural. Sociedades precolombinas suradinas (ed. por V. Williams, B. Ventura, A. Callegari y H. Yacobaccio), pp. 203-219. Artes Gráficas Buschi, Buenos Aires

Scattolin, M.C., Bugliani, F., Izeta, A.. Lazzari, $\quad$ M.; Pereyra Domingorena, L. y L. Martínez.

2001. Conjuntos materiales en dimensión temporal. El sitio formativo "Bañado Viejo" (Valle de Santa María, Tucumán). Relaciones de la Sociedad Argentina de Antropología XXVI: 167-192.

Somonte, C.

2002 El uso del espacio y la produccion y/o descarte de materiales líticos en la Quebrada de Amaicha del Valle, Pcia. de Tucumán. Tesis de Grado en Arqueología. Facultad de Ciencias Naturales e Instituto Miguel Lilo, Universidad Nacional de Tucumán. Tucumán.

2009. Tecnología lítica en espacios persistentes de Amaicha del Valle (Tucumán). Tesis Doctoral en Arqueología. Facultad de Filosofía y Letras, Universidad de Buenos Aires.

Srur, G.

2009. Estudio zooarqueologico en el Sitio Cueva de los Corrales 1 (Quebrada de los Corrales, Tafi del Valle, Pcia de Tucuman).VIII Jornadas de Jóvenes Investigadores en Ciencias Antropológicas, Buenos Aires, 2 al 6 de Noviembre de 2009.

Tartusi, M.R. y V.A. Núñez Regueiro

1993. Los centros ceremoniales del NOA. Publicaciones del Instituto de Arqueología 5, Serie: Ensayo y Crítica 1, Tucumán. 